\title{
The Role of Laser in Infertility Surgery
}

DOI: http://dx.doi.org/10.5915/22-2-13559

\author{
Jamil A. Fayez, M.D. \\ Winston-Salem, North Carolina
}

\begin{abstract}
In the last two decades, developments in gynecologic endoscopy have had an impact on our specialty that is equaled only by the introduction of sophisticated radioimmunoassays. With the exception of dilation and curetage ( $D \& C)$, laparoscopy has become the most frequently performed gynecologic procedure by obstetricians and gynecologists. Although originally introduced as a diagnostic procedure, not suprisingly and within a short time it became an operative procedure. As time went by, more sophistication in operative laparoscopy was obtained because of the development of modern optics and better operative instruments. The latest tool introduced to operative laparoscopy was the laser. This article will discuss all laser laparoscopic procedures that should be done in the field of reproductive surgery.
\end{abstract}

Key words: Laser, laparoscopy, infertility surgery

Since its introduction to the gynecologists in the sixties, laparoscopy has become an integral part of gynecologic surgery for the diagnosis and treatment of abdominal and pelvic diseases of the female reproductive organs. ${ }^{1}$ The $\mathrm{CO}_{2}$ laser has been a feature of intra-adbominal surgery since the early seventies and has been used with laparoscopy since the early eighties. Recent advances in endoscopic surgical techniques and the increased sophistication of surgical instruments have offered new operative methods and techniques for the gynecologic surgeon.

At present, lasers have been used extensively by a small number of reproductive surgeons. Although a large amount of data has been generated, carefully controlled studies are few. Because of the expense of laser equipment, the variety of lasers available, the need for specialized training and the occasional serious complications, the results of ongoing research

From the Department of Obstetrics and Gynecology

Section on Reproductive Endocrinology

Bowman Gray School of Medicine

Wake Forest University

Reprint Requests: Dr. Jamil A. Fayez, M.D.

Department of Obstetrics and Gynecology

Section on Reproductive Endocrinology

Bowman Gray School of Medicine

Wake Forest University

300 South Hawthorne Road

Winston-Salem, North Carolina 27103 are needed before the real role of these instruments is known. Therefore, at present we should accept the surgical laser as a tool, not a panacea. Laser techniques lend themselves to coagulation, ablation and excision. This article is an overview of the $\mathrm{CO}_{2}$ laser and its applications in reproductive surgery.

\section{Techniques and procedures}

All procedures are performed under general endotracheal anesthesia with the patient in the lithotomy position. The bladder is drained and a Foley catheter is left in place throughout the procedure. An intracervical cannula is used for manipulation of the uterus and intraoperative injection of dilute indigo carmine to test tubal patency.

After pneumoperitoneum is established, the operating laparoscope is inserted infraumbilically. After adequate visualization of pelvic organs, two suprapubic puctures are made with $5.5 \mathrm{~mm}$ trocar. The punctures are $4 \mathrm{~cm}$ above the symphysis pubis and $4 \mathrm{~cm}$ from the midline on each side. A probe made of titanium is inserted through one of these trocar sheaths and a suction-irrigation cannula made of titanium is introduced through the second suprapubic puncture site. The only irrigation fluid I use is Ringer's lactate because it has been shown that other adjuvants offer no advantage in preventing postoperative adhesions. ${ }^{2}$

Other ancillary instruments that should be available to the laser laparoscopist include 
atraumatic grasping forceps to stabilize or handle structures such as tubes, ovaries or bowel. Large pieces of tissues may be removed endoscopically by using a long smooth grasping forceps or one with strong teeth, inserted through the operating channel of the $10 \mathrm{~mm}$ operative laparoscope. Large clots may be removed by using a large suction cannula inserted through one of the suprapubic punctures or through the channel of the operating laparoscope.

An 18 gauge needle is inserted through one of the suprapubic punctures for drainage of ovarian cysts, and one with 22 gauge is used to inject dilute vasopressin to produce vasoconstriction. Hemostasis can be achieved either by laser coagulation if the bleeder is less than $0.5 \mathrm{~mm}$ in diameter, or by bipolar electrocautery in case of larger bleeders.

Endoscopic laser have been developed and continuously improved over the past ten years. $\mathrm{A} \mathrm{CO}_{2}$ laser will penetrate a column of water to a depth of only $0.3 \mathrm{~mm}$. Because of very little scattering of the beam, its power can be focused with minimal energy loss to a small point, thus achieving greater energy density, faster heating and faster tissue vaporization. Vaporization occurs by instantaneous boiling of the cellular water at $100^{\circ} \mathrm{C}$. Therefore, unlike other lasers, the $\mathrm{CO}_{2}$ laser can be used for cutting tissue. The extent of damage to adjacent tissue when a scalpel is used is less than with the $\mathrm{CO}_{2}$ laser, but the laser causes less necrosis, inflammation and foreign body reaction than does cautery. ${ }^{3,4}$

A video camera enables the entire surgical team to view the procedure on the videomonitor, producing better coordination between members of the team. The video equipment gives a panoramic and magnified view of the abdominal cavity from many angles. Cameras have become less cumbersome over the past several years. Today one can use a camera that weighs as little as 1.77 ounces and obtains excellent pictures.

\section{Advantages and disadvantages}

The prime advantage of laser procedures done via the operative laparoscope is precise control of the impact zone because the direction of the laser beam and the operator's view coincide. Through endoscopy, the laser allows experienced gynecologists to shift surgery from a major, inpatient operation to a minor, outpatient procedure that demands less time and expense. Laser can reduce the operating time by providing excellent hemostasis. Generally there is less postoperative pain due to minimal tissue damage because of reduced thermal effect on adjacent normal tissue. The smoke and occasional misalignment of the Helium Neon ( $\mathrm{HeNe}$ ) beam are the two major disadvantages of the $\mathrm{CO}_{2}$ laser.

Laparoscopic procedures in which the laser may be employed include ablation of endometriosis, adhesiolysis, uterosacral ablation, tuboplasty, treat- ment of ectopic pregnancy, ablation of hydatid cysts, ablation of fibroids, oophorotomy, and ovarian cystectomies. Each of the procedures will be discussed briefly.

Endometriosis: If there is one place in reproductive gynecologic surgery where the laser is truly valuable, it is in the treatment of endometriosis, where it has proven to be a cost-effective and clinically efficacious technique.' A focused beam of 5,000-20,000 W/ $\mathrm{cm}^{2}$ power density is used to remove or vaporize endometriosis implants from the bladder flap, cul-desac, pelvic sidewalls, ovaries and tubes. Recent data have shown that laser laparoscopy for infertility secondary to endometriosis produced results better than does laparotomy. ${ }^{6}$

For the treatment of endometriomas, most laser laparoscopists open the cyst and remove the capsule of endometrioma. After the capsule is stripped from the ovary, the base is lasered to seal tiny blood vessels and to ensure complete ablation of endometriosis.? No sutures are used to approximate the endometrioma edges because they cause adhesion formation.?

For many years, I have been treating endometriomas by simple drainage, thorough washing and suction, with excellent results. ${ }^{5}$ Recently I started lasing the base of the endometrioma to ensure complete elimination of intracavitary endometriosis. Endometriomas are aspirated first to reduce the amount of spillage of their contents. Then an incision is made in the most dependent portion of the mass to permit the sides of the remaining ovarian tissue to fall together at the conclusion of the procedure. Any residual disease or tiny bleeding points within the ovary are vaporized by laser. The defect need not be closed.

Adhesiolysis: Lysis of abdominal or pelvic adhesions can be effected by using laparoscopic scissors, electrocautery or laser. The adhesions must be stretched to facilitate their excision. ' The main benefits of laser for adhesiolysis are more precise excision of adhesion and minimized bleeding because of its hemostatic effects. In contrast to cautery, adhesiolysis is suited ideally to the $\mathrm{CO}_{2}$ laser because the zone of injury is very narrow. Thus, adhesions involving the tubes, ovaries, uterus or bowel may be approached more aggressively than with cautery. A probe made of titanium should be used as a backstop and placed immediately behind the adhesions being cut in order to absorb the remaining laser energy. Because fluid can act as a large and efficient backstop, an additional safety measure is to maintain a pool of Ringer's lactate in the cul-de-sac.

Uterosacral Ablation: Laser ablation of the uterosacral ligaments in order to relieve dysmenorrhea has been advocated for patients with and without endometriosis." Using the laser, the surgeon can create a crater at the attachment of the ligament 
to the cervix, thus interrupting the parasympathetic and sympathetic sensory nerve fibers to the uterus and cervix (The Frankenhauser Plexus). One study showed significant relief in $81 \%$ of 176 patients. ${ }^{9}$ In patients who get relief, the benefit is usually shortlived and pain may recur in 12 months. Hemorrhage and ureteral injury have been reported and thus caution is mandatory.' The surgeon should exercise particulr care not to cut lateral to the uterosacral ligament where the ureter is located and where the uterosacral branch of the cervical artery courses along its lateral aspect.

Tuboplasty: The blocked distal end of the tube can be opened laparoscopically by scissors or by laser. Tubes that are not densely adherent to ovaries, bowel and/or cul-de-sac are amenable to treatment with $\mathrm{CO}_{2}$ laser via laparoscopy. A cruciate incision is made with a tightly focused beam using super pulse with high power density. I prefer to make this cruciate incision with scissors inserted through the operating channel of the laparoscope. Dilute vasopressin is injected into the mesosalpinx near the distal end of the tube which is already distended with indigo carmine injected via the intracervical cannula. Distension of the tube is essential to identify the dimple which marks the optimal site for opening the tube. After the tube has been opened a low power beam is aimed at the serosal edges to roll them back (flowering technique) and maintain them in an everted position around the newly-created stoma. Pregnancy rate following this laser procedure was reported to vary from $6 \%$ to $36 \%$. $^{10-11}$ In general, patients who become pregnant have less damaged tubes and few peritubal adhesions.

Phimosed tubes can be dilated gently with the grasping forceps and the agglutinated edges are separated and trimmed with high power density laser beam using super pulse mode. I have 27 patients with bilateral tubal phimosis who were treated with laser laparoscopy; $55 \%$ of them achieved intrauterine pregnancy.

Ectopic pregnancy: Since the advent of highresolution ultrasound, using transvaginal transducers combined with the rapid sensitive radioimmunoassays for beta human chorionic gonadotropin, the diagnosis of ectopic pregnancy before its rupture has become the rule rather than the exception. Consequently, most unruptured and many ruptured tubal pregnancies can be treated conservatively using the laparoscope.

At laparoscopy, the site of pregnancy is identified and a dilute 1:50 solution of vasopressin is injected immediately beneath the pregnancy. The tube is stabilized by the atraumatic grasping forceps and a linear incision is made by laser on the antimesenteric side of the tubal segment containing the pregnancy. The pregnancy usually is seen extruding spontaneously and can be pulled out using the grasping forceps. A suction irrigation cannula is utilized to ensure complete evacuation of the pregnancy and to verify that hemostasis is adequate. Rarely, a small vessel will continue to bleed, which can be easily coagulated by laser or cautery. Linear salpingostomy via operative laparoscopy was reported to be safe and effective resulting in future intrauterine pregnancies in $60 \%$ of the patients. ${ }^{12}$ If linear salpingostomy is not feasible and future pregnancy is still desired, segmental excision can be made which allows for future microsurgical anastomosis of the remaining segments of the tube. If pregnancy has ruptured and the tube is beyond repair, or when future pregnancy is not desired, salpingectomy via laparoscope may be done.

Uterine fibroids: Like hydatid cysts and small ovarian fibromas, small subserosal uterine fibroids up to $3 \mathrm{~cm}$ in size may be easily vaporized or excised via laser laparoscopy. Large pedunculated fibroids may be removed by first injecting the base of the stalk with 1:50 dilute vasopressin solution. The stalk is then transected with laser using $15,000 \mathrm{~W} / \mathrm{cm}^{2}$ power density in the continuous mode. The fibroid can be removed by morcellation either via the laparoscopic sheath or through the cul-de-sac via the vagina. Subserosal fibroids with significant intramural components should not be handled via laparoscopy. The risks of hemorrhage, infection and adhesion formation are real, therefore such tumors should be removed via laparotomy.

Oophorotomy for Polycystic ovaries (PCO): Daniell $^{13}$ treated with laser laparoscopy 85 patients with PCO who failed to respond to Clomid therapy. Seventy-one percent of these patients ovulated spotaneously immediately postoperatively and all but one the remaining patients ovulated in response to subsequent Clomid treatment. The ovary is held at its ligament with an atraumatic $5 \mathrm{~mm}$ grasping forceps for proper fixation and adequate exposure of the ovarian surfaces. The $\mathrm{CO}_{2}$ laser is then used to drill holes about $2-4 \mathrm{~mm}$ deep and $3 \mathrm{~mm}$ wide, as judged by the size of the tip of the accessory probe. The number of vaporization sites varies from 25 to 40 depending on the size of the ovary.

I have different results with my patients and therefore I warn against accepting this "drilling" procedure as effective before long term studies are undertaken. The probability of periovarian adhesion formation is high, which would reduce the pregnancy rate in these patients.

Ovarian cystectomy: Ovarian cysts in women in the reproductive age group are largely benign. The overall cancer risk in those between 30 and 35 years of age is estimated to be 4.5 in $200,000 .^{14}$ Cunanan, et $\mathrm{al}^{15}$ reported on laparoscopic aspiration of unilateral unruptured ovarian cysts in 48 patients under the age of 35 , with no intra- or postoperative complications. I usually drain unilocular small 
ovarian cysts with no further therapy except lasing the base to avoid recurrence. However, if the cyst is 5 $\mathrm{mm}$ or more, excision of the cyst wall is essential for pathologic study and to avoid recurrence. The cyst wall can be easily excised by laser or scissors and removed after morcellation via any of the puncture sites.

\section{Conclusion}

The numerous applications of laser laparoscopy have been reviewed and some of the techniques employed have been discussed. It is evident that this type of operative laparoscopy, if appropriately employed, offers numerous advantages, among which are avoiding laparotomy, shortening hospital stay, causing minimal discomfort, reducing health care, and the results are satisfactory.

\section{References}

1. Fayez JA. An assessment of the role of operative laparoscopy in tuboplasty. Fertil Steril. 1983;39:476-479.

2. Fayez JA, Schneider PJ. Prevention of pelvic adhesion formation by different modalities of treatment. Am J Obstet Gynecol. 1987;157:1184-1188.

3. Fayez JA, McComb JS, Harper MA. Comparison of tubal surgery with $\mathrm{CO}_{2}$ laser and the unipolar microelectrode. Fertil Steril. 1983;40:476-480.

4. Bellina JH, Hemmings R, Voras JI, et al. $\mathrm{CO}_{2}$ laser and electrosurgical wound study with an animal model: A comparison of tissue damage and healing patterns in peritoneal tissue. Am J Obstet Gynecol. 1984;148:327-334.

5. Kelly RW, Roberts DK. $\mathrm{CO}_{2}$ laser laparoscopy:
Potential alternative to danazol in the treatment of stage I and II endometriosis. J Reprod Med. 1983;28:638-640.

6. Martin DC. $\mathrm{CO}_{2}$ laser laparoscopy for endometriosis associated with infertility. J Reprod Med. 1986;31:1089-1094.

7. Nezhat C, Winer W, Crowgey S. Videolaseroscopy for treatment of endometriosis and other diseases of the reproductive organs. Obstet Gynecol Forum. 1987;1:1.

8. Fayez JA, Collazo LM. Comparison between laparotomy and operative laparoscopy in the treatment of moderate and severe stages of endometriosis. Int J Fertil (in press).

9. Feste JR. $\mathrm{CO}_{2}$ laser neurectomy for dysmenorrhea. Laser Surg Med. 1984;3:27-30.

10. Daniell JF, Herbert CM. Laparoscopic salpingostomy utilizing the $\mathrm{CO}_{2}$ laser. Fertil Steril. 1984;41:558-563.

11. Nezhat C. Microsurgical treatment of hydrosalpinges and peritubal and periovarian adhesion via videolaseroscopy. Abstract, 43rd Annual Meeting, Am Fertil Steril Society, 1987.

12. Cartwright PS, Herbert CM, Maxon WS. Operative laparoscopy for the management of tubal pregnancy. J Reprod Med. 1986;31:589-591.

13. Daniell JF, Miller W. Polycystic ovaries treated by laparoscopic laser vaporization. Fertil Steril. 1989;51:232-236.

14. Cancer incidence and mortality in the United States. SEER, 1973-81. NIH Publ No. 58-1837. Bethesda, National Institutes of Health, 1984.

15. Cunanan RG, Courey NG, Lippes J. Laparoscopic findings in patients with pelvic pain. Am J Obstet Gynecol. 1983;146:589-591. 